\title{
Hydroformylation in reverse micellar systems
}

\author{
Frédéric Van Vyve*, Albert Renken \\ Swiss Federal Institute of Technology Lausanne (EPFL), Lausanne, Switzerland
}

\begin{abstract}
Different micellar systems are characterized. Hydroformylation reactions are carried out in the most appropriate ones and are compared with hydroformylations in a non-micellar system. Reaction rates and selectivities for aldehydes are higher than the micelles. In addition to this, olefins between $\mathrm{C}_{6}$ and $\mathrm{C}_{16}$ are successfully hydroformylated in the micellar system, whereas $\mathrm{C}_{8}$ is the highest olefin that can be hydroformylated without micelles. The behavior of the reaction in the micellar system is very sensitive to the initial composition and reaction conditions. (C) 1999 Elsevier Science B.V. All rights reserved.
\end{abstract}

Keywords: Microemulsion; Hydroformulation; Inverse micelles; Water-soluble rhodium complex

\section{Introduction}

The hydroformylation of olefins, also known as oxo synthesis, was discovered in 1938 by Roelen at Ruhrchemie. Olefins react with carbon monoxide and hydrogen in the presence of a catalyst to form aldehydes containing an additional carbon atom. Presently the total worldwide oxo production capacity for aldehydes is about $6.10^{6}$ t/year [1].

$$
\begin{aligned}
& \mathrm{R}-\mathrm{CH}=\mathrm{CH}_{2} \underset{\text { cat }}{\stackrel{\mathrm{CO}, \mathrm{H}_{2}}{\longrightarrow}} \mathrm{R}-\mathrm{CH}_{2}-\mathrm{CH}_{2}-\mathrm{CHO}, \\
& \mathrm{R}-\mathrm{CH}-\mathrm{CHO} \\
& \quad \mid \\
& \mathrm{CH}_{3}
\end{aligned}
$$

Besides the desired $n$-aldehyde, the formation of iso-aldehyde in a parallel reaction must be considered. The Ruhrchemie/Rhône-Poulenc (RCH-RP) process is a low pressure (40-60 bar) gas-liquid-liquid pro-

\footnotetext{
*Corresponding author.
}

cess based on a water-soluble rhodium complex catalyst [2]. The use of a water-soluble complex brings with it substantial advantages for industrial practice, because the catalyst can be considered to be heterogeneous. Since the catalyst is insoluble in the organic phase formed, losses of the noble metal in the crude aldehyde are negligible. High-boiling by-products do not dissolve in the aqueous catalyst phase, thus making catalyst regeneration irrelevant. Unfortunately, this process is mainly limited to short chain olefins $\left(\mathrm{C}_{3}-\mathrm{C}_{6}\right)$ since the reaction rate drops dramatically for longer alkenes, which can only be hydroformylated in homogeneous systems under more severe conditions.

\section{Theory}

This project, carried out in collaboration with Hoechst AG, consists in the development of an alternative to the RCH-RP process for the hydroformylation of olefins. The studied alternative applies reverse micelle technology, mainly used in biotechnology, to 
catalytic hydroformylation. This consists in adding an appropriate amphiphilic agent (surfactant or detergent) to the biphasic liquid system, so that the aqueous and organic phases form a single stable micellar medium or a microemulsion, with continuous and dispersed phases.

Amphiphilic molecules are composed of a hydrophobic part (hydrocarbon chain) and a hydrophilic part. The hydrophilic part is called the head group and may be dipolar (zwitterionic), ionic (cationic or anionic) or non-ionic. This combination of pronounced hydrophobic and hydrophilic properties within one molecule confers unique properties to these amphiphiles upon dissolution in water. At very low concentrations, the ionic amphiphiles with a hydrocarbon chain behave as normal electrolytes, but if their concentration is raised above the so-called critical micellar concentration (CMC), aggregates (micelles) are formed (Fig. 1). If the CMC is considered to be the solubility limit of the monomeric agent, it is the micellization process which prevents precipitation of the amphiphile. This co-operative association starts in a narrow concentration range and is revealed by rather abrupt changes in a variety of physical properties such as conductivity, surface tension, viscosity, and self-diffusion coefficient [3].

Working in reverse micellar phases thus presents two major advantages. Firstly, micelles can dissolve molecules which are normally not soluble in the bulk solvent [4]. Secondly, due to the very small size of micelles $(10-100 \mathrm{~nm})$, there is an enormous increase of the interface between the continuous and the dispersed pseudo-phases in comparison to a mechanically stirred system without surfactant.

On the other hand, since the micellar phase is stable, a method must be developed to separate the two phases at the end of the reaction, in order to recover the aqueous catalytic phase for a further reaction.

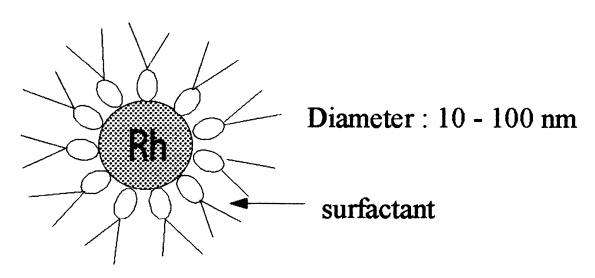

Fig. 1. Schematic representation of an reverse micelle.
A first successful attempt at hydroformylation in a micellar phase was made by Tinucci and Platone [5], who showed in a patent that olefins could be hydroformylated with satisfactory reaction rates and selectivities. The micellar system chosen was a mixture of sodium dodecyl sulfate (surfactant) and butanol (cosurfactant).

\section{Objectives}

The main objectives of this work are:

1. The characterization of different micellar systems and the choice of the most appropriate one for the hydroformylation reaction.

2. A study of the effects of different parameters on the behavior of the reaction in the micellar system and a comparison with results obtained in the nonmicellar mode.

\section{Methods}

All hydroformylation reactions were carried out in a 0.51 stainless steel autoclave (Büchi). Temperature was regulated by an oil bath (Haake N3) and the reactor operated in semi-batch mode with synthesis gas being permanently introduced to maintain a constant pressure and to compensate gas consumption due to the reaction. Samples were taken at intervals and analyzed by gas chromatography (Perkin-Elmer Autosystem). Reproducibility was ensured by carrying out each reaction at least twice.

The reactions were carried out at $125^{\circ} \mathrm{C}$ and under a pressure of 30 bar of synthesis gases, composed of a mixture of hydrogen and carbon monoxide in a ratio $1: 1$. The catalyst precursor, rhodium acetate, was first pre-treated during $3 \mathrm{~h}$ in the presence of water and ligand $(\mathrm{TPPTS}=$ tri-sulfophenylphosphine as sodium salt, see Fig. 2(a)) at $125^{\circ} \mathrm{C}$ and under 30 bar of synthesis gas, to form the catalytic complex (Fig. 2(b)). After this treatment, the olefin and the surfactant were added from an auxiliary tank to start the reaction.

In all cases, except for experiments presented in Fig. 4, the composition chosen for the hydroformylations in the micellar system was 60 mass percent of 
<smiles>NS(=O)(=O)c1cccc(P(c2cccc(S(N)(=O)=O)c2)c2cccc(S(=O)(=O)O[Na])c2)c1</smiles><smiles>[2H][PH2+](c1ccccc1)[PH]([PH2+])([PH2+])C=O</smiles>

Fig. 2. (a) The TPPTS ligand and (b) The catalytic complex.

organic phase, $25 \%$ of surfactant and $15 \%$ of aqueous phase, as shown in Fig. 3. For an easier comparison, the same proportions were kept for systems without micelles. In the absence of surfactant, the composition was 80 mass percent of organic phase and $20 \%$ of aqueous phase. The system was also defined by two ratios: the molar ratio between olefin and catalyst and the molar ratio between ligand and catalyst.
The non-micellar composition is therefore very different with respect to the organic to aqueous phase volume ratio of 1:2 described for the RCH-RP process. The later also uses a much higher ligand:Rh ratio. Nevertheless, the reaction rates observed for the two processes are similar.

\section{Results and discussion}

\subsection{Choice of micellar system}

Twelve micellar systems were investigated alone and in the presence of co-surfactants, with regard to their ability to form reverse micelles that contain a large amount of dispersed aqueous phase containing the catalyst and their stability under reaction conditions (above $100^{\circ} \mathrm{C}$ ) with a wide variety of olefins and ranges of temperatures. The characterization was done by determining the limit of stability of these micellar systems on ternary diagrams at different temperatures.

Ethoxylates, $\mathrm{C}_{11}$-oxoalcohols and alkylphenols containing various numbers of ethylene oxide units (Arkopal), are soluble in the organic phase only in very small amounts and incorporate no water, even

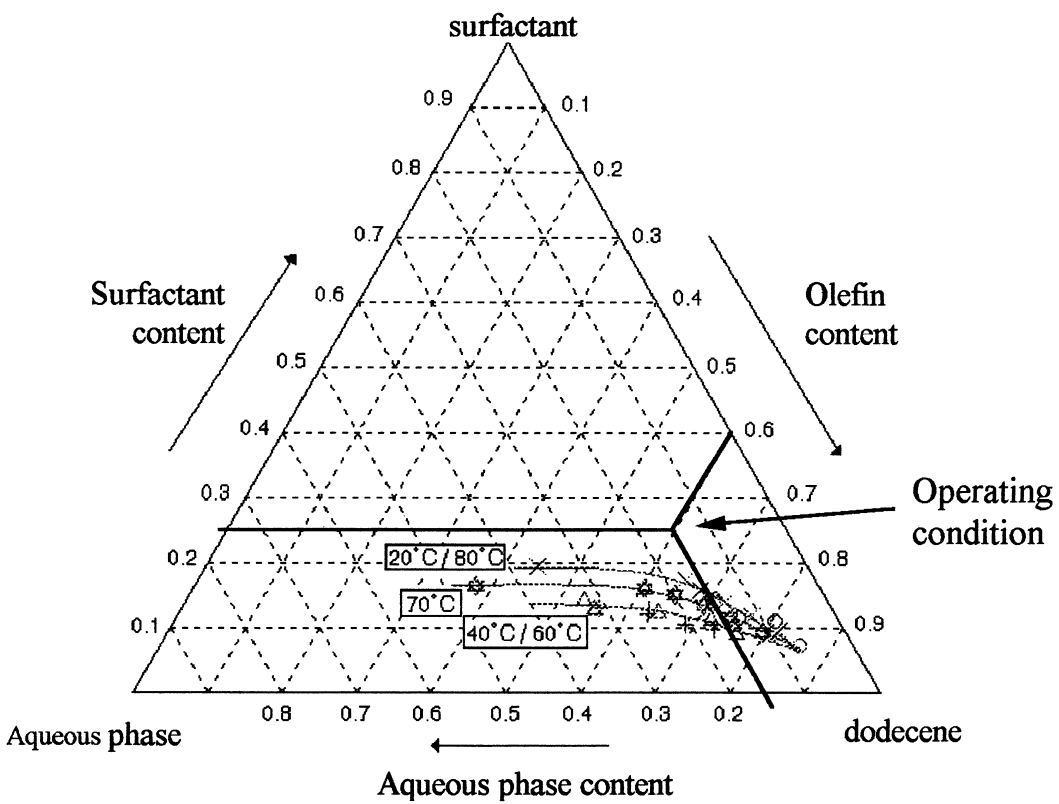

Fig. 3. Ternary diagram for the system dodecene/surfactant/water. 
with butanol or Flotanol ${ }^{\circledR}$ (mixture of polyglycols and ethers) as a co-surfactant. Ethylene oxide/propylene oxide block copolymers (Genapol) containing up to $80 \%$ of ethylene oxide functions form a thick gel when water is added, even in the presence of co-surfactants.

Sodium dodecylsulfate (used by Tinucci and Platone [5]), sodium dioctyl sulfosuccinate and an alkane sulfonate, in association with butanol, matched the desired criteria and were found suitable for the hydroformylation reactions. The ternary diagram constructed with the chosen surfactant ${ }^{1}$, for the system dodecene/surfactant/water, is presented as an example in Fig. 3 between $20^{\circ} \mathrm{C}$ and $80^{\circ} \mathrm{C}$. Since the characterization is done under atmospheric pressure in glass vessel, higher temperatures cannot be reached.

\subsection{Influence of the composition of the reaction mixture on reaction kinetics}

As a consequence of the stability limits of the micelles, the reaction system must be chosen with the volume ratio between the organic and the aqueous catalytic phases as a constraint. The micellar system is therefore defined by three parameters: a composition chosen from the diagram defined by an olefin/surfactant/aqueous phase mass ratio, an olefin/catalyst molar ratio and a ligand/catalyst molar ratio. The effect of the following parameters on the reaction behavior are investigated:

- ligand to catalyst molar ratio from 4:1 to $20: 1$,

- olefin to catalyst molar ratio from 1000:1 to 5000:1,

- organic to aqueous phase volume ratio from 2:1 to $4: 1$, and

- surfactant mass percentage in the mixture from $25 \%$ to $35 \%$.

All these parameters have an important impact on the behavior of the hydroformylation, modifying the reaction rate, the selectivity towards aldehydes (a parallel isomerization of the 1-alkene to 2-alkene being the main undesirable reaction) or the $n$ :iso ratio between linear and branched aldehydes. As an example, Fig. 4 and Table 1 represent the results of the

\footnotetext{
${ }^{1}$ This paper is presently submitted to our industrial partner and the nature of the chosen surfactant will be revealed with his agreement.
}

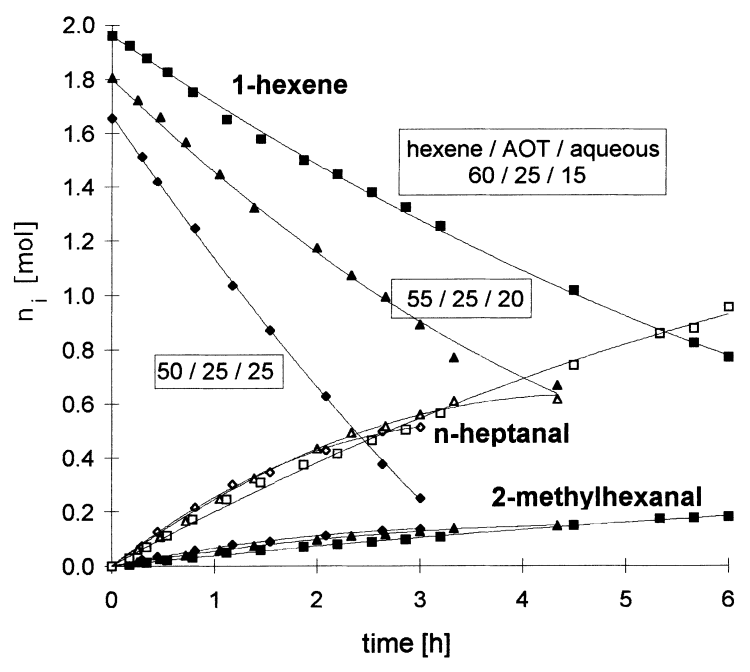

Fig. 4. Effect of initial composition on the hydroformylation of hexene in the microemulsion.

Table 1

Effect of initial composition on the hydroformylation of 1-hexene in the microemulsion

\begin{tabular}{llll}
\hline & $60 / 25 / 15$ & $55 / 25 / 20$ & $50 / 25 / 25$ \\
\hline$-\mathrm{R}_{0, \text { alkene }}\left(\mathrm{mol} \mathrm{h}^{-1} \mathrm{~mol}_{\mathrm{Rh}}^{-1}\right)$ & 226 & 372 & 574 \\
$\mathrm{~S}_{0, \text { aldehydes }}$ & 0.95 & 0.73 & 0.53 \\
$\mathrm{~S}_{0, n \text {-aldehyde }}$ & 0.80 & 0.60 & 0.42 \\
$n:$ iso ratio & $84: 16$ & $82: 18$ & $79: 21$ \\
\hline
\end{tabular}

hydroformylation of 1-hexene with three different initial organic/surfactant/aqueous compositions, where the surfactant proportion remains constant.

The results (Fig. 4) clearly show that increasing the ratio between organic and aqueous phases (with a constant surfactant proportion of 25 mass percent) has no effect on the production rate of aldehydes, whereas the consumption rate of the alkene diminishes. This modification is due to an important diminution of the isomerization of the 1-alkene into 2alkene, which leads to a significant increase in the selectivity towards aldehydes. This is confirmed in Table 1.

The reaction rates are estimated by extrapolating to the origin, a second order equation that fits the measured points. The selectivity for the total aldehydes, respectively, for the straight-chain aldehyde, is obtained by dividing the production rate of the alde- 

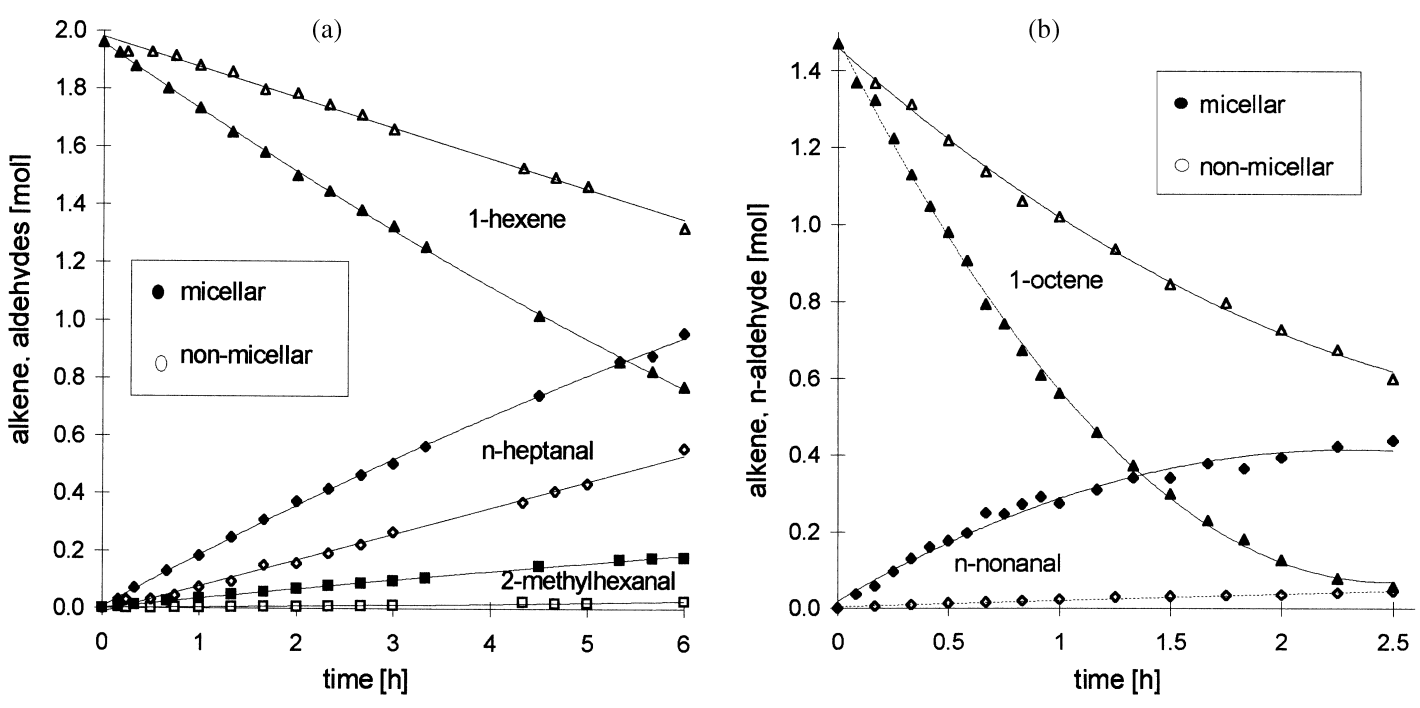

Fig. 5. Comparison of the hydroformylation of hexene and octene in micellar and non-micellar systems.

hydes, respectively, of the straight-chain aldehyde, by the consumption rate of the alkene. Thus, the calculated data are initial reaction rates $\left(-\mathrm{R}_{0, \text { olefine }}\right)$ and instantaneous initial selectivities $\left(\mathrm{S}_{0, \text { aldehydes}}\right.$, respectively $\mathrm{S}_{0, n \text {-aldehyde) }}$.

\subsection{Comparison of hydroformylation in micellar and non-micellar systems}

As the non-micellar process is known to be efficient only for short-chain olefins, this study is divided into two parts:

1. Hexene, octene and decene are hydroformylated in micellar and non-micellar systems.

2. Longer olefins, up to hexadecene, are hydroformylated only in the micellar system.
Fig. 5(a) and (b) show the consumption of the olefin and the production of straight-chain aldehyde for the hydroformylations of 1-hexene and 1-octene in micellar and non-micellar modes.

Since the reaction rate of 1-decene in the nonmicellar system is extremely slow, it is not shown graphically, but only reported in the comparative Table 2.

A much lower rate of production of aldehydes is observed with increasing chain length of the olefin in the non-micellar system, this rate proportional is to the solubility in the aqueous phase as described by Bogdanovic [6]. By contrast, there is no clear relationship between the reaction rate or selectivity and olefin chain length in the micellar mode, with values consistently higher than those for the non-micellar process. This indicates that the rate is controlled, not only

Hydroformylation of several olefins in micellar and non-micellar systems

\begin{tabular}{|c|c|c|c|c|c|c|}
\hline & \multicolumn{2}{|l|}{ 1-Hexene } & \multicolumn{2}{|l|}{ 1-Octene } & \multicolumn{2}{|c|}{ 1-Decene } \\
\hline & Micellar & Non-micellar & Micellar & Non-micellar & Micellar & Non-micellat \\
\hline$-\mathrm{R}_{0, \text { alkene }}\left(\mathrm{mol} \mathrm{h}^{-1} \mathrm{~mol}_{\mathrm{Rh}}^{-1}\right)$ & 226 & 84 & 1531 & 513 & 1003 & 12 \\
\hline $\mathrm{R}_{0, n \text {-aldehyde }}\left(\mathrm{mol} \mathrm{h}^{-1} \mathrm{~mol}_{\mathrm{Rh}}^{-1}\right)$ & 183 & 77 & 568 & 24 & 330 & 2 \\
\hline $\mathrm{S}_{0, \text { aldehydes }}$ & 0.96 & 0.95 & 0.47 & 0.07 & 0.41 & 0.18 \\
\hline $\mathrm{S}_{0, n \text {-aldehyde }}$ & 0.81 & 0.91 & 0.37 & 0.05 & 0.33 & 0.16 \\
\hline$n$ :iso ratio & $84: 16$ & $95: 5$ & $80: 20$ & $75: 25$ & $79: 21$ & 89: 11 \\
\hline
\end{tabular}




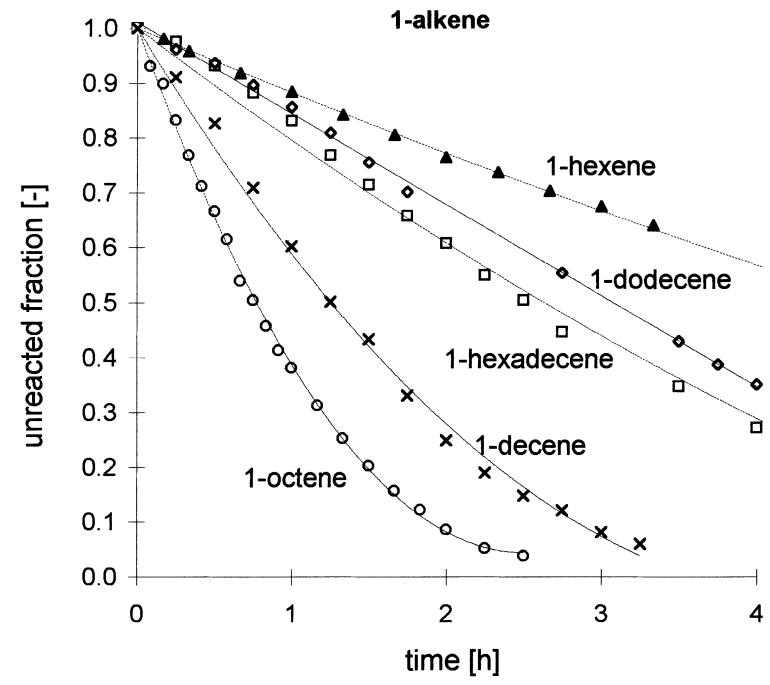

Fig. 6. Consumption of alkene for the hydroformylation of different olefins in the micellar system.

by the solubility of the alkene, but also by micellar stability phenomena. Indeed, the micellar stability (size and shape of the micelles) and behavior of the reaction is strongly influenced both by the type of organic phase (mixture of alkene and aldehyde) and the organic/surfactant/aqueous composition. Thus, the composition used for the hydroformylation of the different olefins in Table 2 is not necessarily the optimum and may therefore explain the variations in rate and selectivity.

\subsection{Hydroformylation of higher olefins in the micellar system}

Longer olefins are hydroformylated in the micellar mode in order to have a complete series from 6 to 16 carbon chains (hexene to hexadecene).

Fig. 6 shows the consumption of the olefin for the hydroformylation of 1-hexene, 1-octene, 1-decene, 1dodecene and 1-hexadecene. For experimental purposes, the same mass of olefin is used for every hydroformylation and the initial number of moles is thus different for each olefin. To ensure an easier legibility of the figures, the measured values are normalized by the initial number of moles of the olefin. For this reason, Fig. 6 presents the unreacted fraction of each olefin, Fig. 7(a) presents the yield of straight-chain aldehyde and Fig. 7(b) presents the yield of iso-aldehyde.

The reaction rates and selectivities of these reactions are reported in Table 3.

The results show that in an appropriate micellar system interesting reaction rates can be obtained for long-chain olefins which do not react in the nonmicellar mode. The production rate of heptadecanal
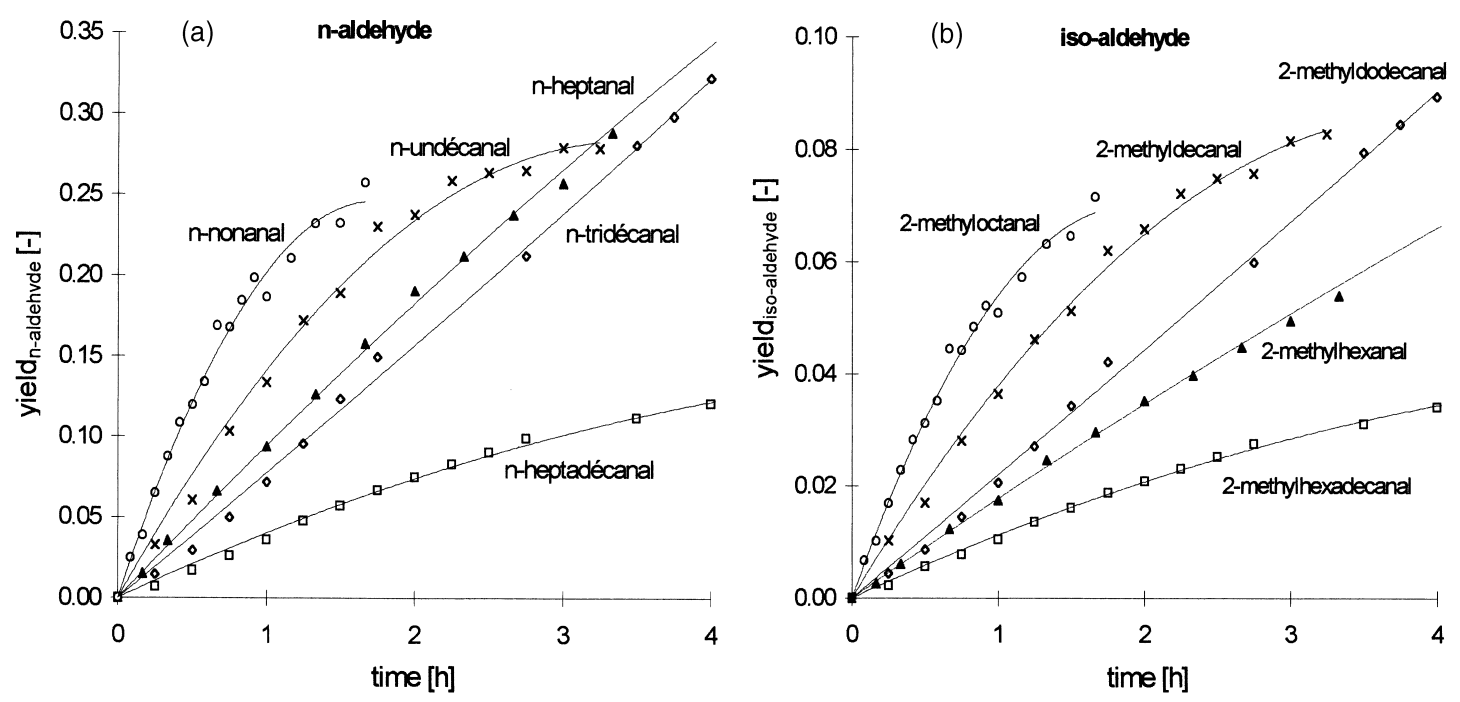

Fig. 7. Production of straight-chain aldehyde and iso-aldehyde for the hydroformylation of several olefins in the micellar system. 
Table 3

Reaction rates and selectivities for the hydroformylation of several olefins in the micellar system

\begin{tabular}{llllll}
\hline & Hexene & Octene & Decene & Dodecene & Hexadecene \\
\hline$-\mathrm{R}_{0, \text { alkene }}\left(\mathrm{mol} \mathrm{h}^{-1} \mathrm{~mol}_{\mathrm{Rh}}^{-1}\right)$ & 226 & 1531 & 1003 & 309 & 493 \\
$\mathrm{R}_{0, n \text {-aldehyde }}\left(\mathrm{mol} \mathrm{h}^{-1} \mathrm{~mol}_{\mathrm{Rh}}^{-1}\right)$ & 183 & 568 & 330 & 152 & 87 \\
$\mathrm{~S}_{0, \text { aldehydes }}$ & 0.96 & 0.47 & 0.41 & 0.63 & 0.22 \\
$\mathrm{~S}_{0, n \text {-aldehyde }}$ & 0.81 & 0.37 & 0.33 & 0.49 & 0.18 \\
$n$ :iso ratio & $84: 16$ & $80: 20$ & $79: 21$ & $78: 22$ & $78: 22$ \\
\hline
\end{tabular}

is even greater than what was obtained for heptanal without micelles.

Except for the hydroformylation of hexene, i.e. from nonanal to heptadecanal, the production rate of straight-chain aldehyde decreases at an approximate rate of $24 \%$ for each additional carbon atom in the olefin chain. On the other hand, no correlation between the chain length and the consumption rate of the alkene can be drawn. This is due to important variations in the selectivities for the different aldehydes, which can be explained by the modifications of stability of the micelles depending on the transformed alkene.

\section{Conclusions and perspectives}

The use of a micellar system for the heterogeneous catalytic hydroformylation of olefins under mild conditions is a very efficient alternative to the existing biphasic process.

High reaction rates can be obtained with good selectivity for the desired aldehydes. It is even possible to hydroformylate long-chain olefins (up to 16 carbon atoms at least) which could only be hydroformylated in homogeneous systems under high pressure.

On the other hand, the behavior of a micellar phase (stability, size and shape of the micelles) is rather complex and depends on many parameters that must still be optimized to obtain an optimal selectivity for the desired aldehydes. For example, the changes in medium composition during the course of the reaction modifies its structure and may even lead to phase separation.

An important aspect of the micellar hydroformylation is the recovery of the aqueous phase containing the catalyst at the end of the reaction. Different processes are considered and their experimentation is presently in progress.

\section{Acknowledgements}

Financial support of the Hoechst AG, Frankfurt, is gratefully acknowledged.

\section{References}

[1] H. Bahrmann, H. Bach, Ullmann A18 (1990) 321-327.

[2] E. Wiebus, B. Cornils, Chem. Ing. Technol. 66(7) (1994) 916-923.

[3] E. Sudhölter, G. van de Langkruis, J. Roy. Netherlands Chem. Soc. 99(3) (1980) 73-82.

[4] R. Schömacker, Nachr. Chem. Tech. Lab. 40(12) 1992.

[5] L. Tinucci, E. Platone, ENIRICERCHE S.p.A., European Patent Specification No. 0380154 B1, 1994.

[6] S. Bogdanovic, Private communication, 1996. 